\title{
Analysis of $\Lambda_{c}(2595), \Lambda_{c}(2625), \Lambda_{b}(5912), \Lambda_{b}(5920)$ based on a chiral partner structure
}

\author{
Yohei Kawakami* and Masayasu Harada $^{\dagger}$ \\ Department of Physics, Nagoya University, Nagoya 464-8602, Japan
}

(Received 25 April 2018; published 22 June 2018)

\begin{abstract}
We construct an effective hadronic model including $\Lambda_{c}(2595), \Lambda_{c}(2625), \Lambda_{b}(5912)$, and $\Lambda_{b}(5920)$, regarding them as chiral partners to $\Sigma_{c}(2455), \Sigma_{c}(2520), \Sigma_{b}$, and $\Sigma_{b}^{*}$, respectively, with respecting the chiral symmetry and heavy-quark spin-flavor symmetry. We determine the model parameters from the experimental data for relevant masses and decay widths of $\Sigma_{c}^{(*)}$ and $\Lambda_{c}(2595)$. Then, we study the decay widths of $\Lambda_{c}(2625)$, $\Lambda_{b}(5912)$, and $\Lambda_{b}(5920)$. We find that, although the decay of $\Lambda_{c}(2595)$ is dominated by the resonant contribution through $\Sigma_{c}(2455)$, nonresonant contributions are important for $\Lambda_{c}(2625), \Lambda_{b}(5912)$, and $\Lambda_{b}(5920)$, which reflects the chiral partner structure. We also study the radiative decays of the baryons, and show that each of their widths is determined from the radiative decay width of their chiral partners.
\end{abstract}

DOI: 10.1103/PhysRevD.97.114024

\section{INTRODUCTION}

Chiral symmetry and its spontaneous breaking is one of the most important properties to understand the structures of hadrons including light quarks. The spontaneous chiral symmetry breaking is expected to generate a part of hadron masses and causes mass difference between chiral partners. We expect that the study of chiral partner structure will provide a clue for understanding the chiral symmetry.

In Refs. [1-5], the chiral partner structure of heavy-light mesons was studied regarding the mesons with $J^{P}=$ $\left(0^{+}, 1^{+}\right)$such as $\left(D_{0}^{*}, D_{1}\right)$ as the chiral partners to the mesons with $J^{P}=\left(0^{-}, 1^{-}\right)$such as $\left(D, D^{*}\right)$ based on the chiral symmetry combined with the heavy-quark spin symmetry. In Refs. [6-8], doubly heavy baryons with negative parity were studied by regarding them as chiral partners to the positive parity heavy baryons. In these analysis, the heavy-quark flavor symmetry, in addition to the chiral symmetry and the heavy-quark spin symmetry, plays a very important role to relate the charm baryons to the bottom baryons. In Refs. [9,10], the chiral partner structure of heavy baryons including a charm quark is within the bound state approach based on the Skyrm model. In Ref. [11], the chiral partner structure of single heavy baryons was studied, in which the chiral partner of $\Sigma_{c}$ baryon with positive parity is regarded as the $\Sigma_{c}$ baryons with negative parity.

\footnotetext{
*kawakami@hken.phys.nagoya-u.ac.jp

"harada@hken.phys.nagoya-u.ac.jp
}

Published by the American Physical Society under the terms of the Creative Commons Attribution 4.0 International license. Further distribution of this work must maintain attribution to the author(s) and the published article's title, journal citation, and DOI. Funded by SCOAP .
In the present work, we would like to propose a new possibility of the chiral partner structure for single heavy baryons that is different from the one in Ref. [11], in which the chiral partners of $\Sigma_{Q}(Q=c, b)$ baryons with positive parity are considered as $\Lambda_{Q}$ baryons with negative parity: we regard $\left(\Lambda_{c}\left(2595 ; J^{P}=1 / 2^{-}\right), \Lambda_{c}\left(2625 ; 3 / 2^{-}\right)\right)$as the chiral partners to $\left(\Sigma_{c}\left(2455 ; 1 / 2^{+}\right), \Sigma_{c}\left(2520 ; 3 / 2^{+}\right)\right)$, and $\left(\Lambda_{b}(5912\right.$; $\left.\left.1 / 2^{-}\right), \Lambda_{b}\left(5920 ; 3 / 2^{-}\right)\right)$to $\left(\Sigma_{b}\left(1 / 2^{+}\right), \Sigma_{b}^{*}\left(3 / 2^{+}\right)\right)$. Based on this chiral partner structure, we construct an effective model respecting the chiral symmetry and the heavy-quark spin-flavor symmetry. Determining model parameters from the experimental data for relevant masses and decay widths of $\Sigma_{c}(2455), \Sigma_{c}^{*}(2520)$, and $\Lambda_{c}(2595)$, we study the decay widths of $\Lambda_{c}^{*}(2625), \Lambda_{b}(5912)$, and $\Lambda_{b}(5920)$. The properties of these states have been studied experimentally and theoretically (see, e.g., Refs. [11-29] and references therein). In the present analysis, we compare our predictions of the decay widths with other available predictions by quark models [12,13] and chiral effective models $[14,15]$.

This paper is organized as follows: In Sec. II, we study the chiral structure of single heavy baryons (SHBs). We construct an effective Lagrangian in Sec. III. Sections IV and V are devoted to study the masses and the hadronic decays of SHBs. We also study the radiative decays of SHBs in Sec. VI. Finally, we give a summary and discussions in Sec. VII.

\section{CHIRAL STRUCTURE OF SINGLE HEAVY BARYONS}

In this section, we study the chiral structure of single heavy baryons using interpolating quark fields.

First, we consider interpolating field operators made from up or down quarks: 


$$
q_{L, R}^{i}, \quad(i=u, d),
$$

where $L$ and $R$ denote left-handed and right-handed chirality, respectively. By using these, we can construct two combinations of diquarks carrying spin zero which are expressed as

$$
\left(q_{L}^{i}\right)^{T} C q_{L}^{j}, \quad\left(q_{R}^{i}\right)^{T} C q_{R}^{j},
$$

where ${ }^{T}$ denote the transposition in the spinor space and $C=i \gamma^{0} \gamma^{2}$ is the charge conjugation matrix. When the relative angular momentum between two quarks is even, the indices $i$ and $j$ should be antisymmetrized due to the Fermi statistics. In such a case, we can easily see that both of the above diquarks are chiral singlet. For clarifying this chiral structure, we introduce the following two diquarks which are chiral singlet:

$$
\epsilon_{i j}\left(q_{L}^{i}\right)^{T} C q_{L}^{j}, \quad \epsilon_{i j}\left(q_{R}^{i}\right)^{T} C q_{R}^{j},
$$

where $\epsilon^{i j}$ is antisymmetric tensor, $\epsilon_{i j}=-\epsilon_{j i}$, with $\epsilon_{u d}=1$, and the summations over repeated indices are understood. Since both of the above two diquarks are chiral singlet, two combinations of them, which are parity eigenstates, are separately chiral singlet.

Now, let us introduce a field for the chiral-singlet lightquark cloud with $J^{P}=0^{+}$as

$$
\Phi_{(+)}=\epsilon_{i j}\left(q_{L}^{i}\right)^{T} C q_{L}^{j}+\epsilon_{i j}\left(q_{R}^{i}\right)^{T} C q_{R}^{j},
$$

which belongs to $(1,1)$ representation under $\left(\mathrm{SU}(2)_{L}\right.$, $\left.\mathrm{SU}(2)_{R}\right)$ symmetry. We construct a single heavy baryon by combining this light-quark cloud $\left(J^{P}=0^{+}\right)$to a heavy quark $Q(Q=c, b)$. The resultant baryon is a heavy-quark spin singlet, so that we identify it with the lightest $\Lambda_{Q}$ $(Q=c, b)$ :

$$
\Lambda_{Q} \sim Q \Phi_{(+)}, \quad \Lambda_{Q}=\left(\Lambda_{c}^{+}, \Lambda_{b}^{0}\right),
$$

which belongs to $(1,1)$ representation under $\left(\mathrm{SU}(2)_{L}\right.$, $\left.\mathrm{SU}(2)_{R}\right)$ symmetry.

Next, we consider the following diquark:

$$
\left[\Phi^{\mu}\right]^{i j}=\left[q_{L}^{T} C \gamma^{\mu} q_{R}\right]^{i j}=\left(q_{L}^{i}\right)^{T} C \gamma^{\mu} q_{R}^{j},
$$

which belongs to $(2,2)$ representation under $\left(\mathrm{SU}(2)_{L}\right.$, $\left.\mathrm{SU}(2)_{R}\right)$ symmetry. We can easily see that the following property is satisfied:

$$
\left[q_{R}^{T} C \gamma^{\mu} q_{L}\right]^{i j}=-\left[q_{L}^{T} C \gamma^{\mu} q_{R}\right]^{j i}
$$

From these diquarks, we make two combinations of parity eigenstates:

$$
\begin{gathered}
{\left[q_{L}^{T} C \gamma^{\mu} q_{R}\right]^{i j}+\left[q_{R}^{T} C \gamma^{\mu} q_{L}\right]^{i j}=\left[q^{T} C \gamma^{\mu} q\right]^{i j}=\left[\Phi_{(3)}^{\mu}\right]^{i j},} \\
{\left[q_{L}^{T} C \gamma^{\mu} q_{R}\right]^{i j}-\left[q_{R}^{T} C \gamma^{\mu} q_{L}\right]^{i j}=\left[q^{T} C \gamma^{\mu} \gamma_{5} q\right]^{i j}=\left[\Phi_{(1)}^{\mu}\right]^{i j} .}
\end{gathered}
$$

From the property in Eq. (7), one can easily check that the indices of the diquark with $J^{P}=1^{+}$is symmetric in the light-quark flavor space, and those of the one with $J^{P}=1^{-}$ is antisymmetric, i.e.,

$$
\left[\Phi_{(3)}^{\mu}\right]^{i j}=\left[\Phi_{(3)}^{\mu}\right]^{j i}, \quad\left[\Phi_{(1)}^{\mu}\right]^{i j}=-\left[\Phi_{(1)}^{\mu}\right]^{j i} .
$$

From this we can easily see that, when the chiral symmetry is spontaneously broken into the isospin symmetry, $\Phi_{(3)}^{\mu}$ is the iso-triplet diquark with $J^{P}=1^{+}$, and $\Phi_{(1)}^{\mu}$ is the isosinglet diquark with $J^{P}=1^{-}$.

The diquark $\Phi^{\mu}$ combined with a heavy quark makes a set of heavy-quark doublets of SHBs with $1 / 2^{-}$and $3 / 2^{-}$as

$$
S_{Q}^{\mu} \sim Q \Phi^{\mu},
$$

where $S_{Q}^{\mu}$ denotes the field for the set of SHBs. The $S_{Q}^{\mu}$ includes iso-triplet SHBs and iso-singlet SHBs as

$$
\begin{aligned}
\left(\Sigma_{Q}^{a}\left(1 / 2^{+}\right), \Sigma_{Q}^{* a}\left(3 / 2^{+}\right)\right) & \sim Q \Phi_{(3)}^{\mu}, \\
\left(\Lambda_{Q 1}\left(1 / 2^{-}\right), \Lambda_{Q 1}^{*}\left(3 / 2^{-}\right)\right) & \sim Q \Phi_{(1)}^{\mu},
\end{aligned}
$$

where we omitted the index $\mu$ in the left-hand sides. It should be stressed that, since both $\Phi_{(3)}^{\mu}$ and $\Phi_{(1)}^{\mu}$ are included in one chiral multiplet $\Phi^{\mu}$, the heavy-quark multiplet of $\left(\Lambda_{Q 1}\left(1 / 2^{-}\right), \Lambda_{Q 1}^{*}\left(3 / 2^{-}\right)\right)$is the chiral partner to that of $\left(\Sigma_{Q}\left(1 / 2^{+}\right), \Sigma_{Q}^{*}\left(3 / 2^{+}\right)\right)$. In the present work, we identify $\left(\Sigma_{Q}\left(1 / 2^{+}\right), \Sigma_{Q}^{*}\left(3 / 2^{+}\right)\right)$with the lightest iso-triplet singleheavy baryons with positive parity, and $\left(\Lambda_{Q 1}\left(1 / 2^{-}\right)\right.$, $\left.\Lambda_{Q 1}^{*}\left(3 / 2^{-}\right)\right)$with the lightest iso-singlet ones with negative parity:

$$
\begin{aligned}
\left(\Sigma_{c}, \Sigma_{c}^{*}\right) & =\left(\Sigma_{c}\left(2455 ; 1 / 2^{+}\right), \Sigma_{c}\left(2520 ; 3 / 2^{+}\right)\right), \\
\left(\Lambda_{c 1}, \Lambda_{c 1}^{*}\right) & =\left(\Lambda_{c}\left(2595 ; 1 / 2^{-}\right), \Lambda_{c}\left(2625 ; 3 / 2^{-}\right)\right), \\
\left(\Sigma_{b}, \Sigma_{b}^{*}\right) & =\left(\Sigma_{b}\left(1 / 2^{+}\right), \Sigma_{b}^{*}\left(3 / 2^{+}\right)\right), \\
\left(\Lambda_{b 1}, \Lambda_{b 1}^{*}\right) & =\left(\Lambda_{b}\left(5912 ; 1 / 2^{-}\right), \Lambda_{b}\left(5920 ; 3 / 2^{-}\right)\right) .
\end{aligned}
$$

\section{EFFECTIVE LAGRANGIAN}

In this section we construct an effective Lagrangian for the relevant SHBs based on the heavy-quark spin-flavor symmetry and the chiral symmetry. We use the field $\Lambda_{Q}$ for expressing the SHBs belonging to the chiral singlet in Eq. (5). For expressing the SHBs belonging to chiral $(2,2)$ representations, we introduce the field $S_{Q}^{\mu}$ in Eq. (10) which transforms as 


$$
S_{Q}^{\mu} \stackrel{\mathrm{Ch}}{\rightarrow} g_{R} S_{Q}^{\mu} g_{L}^{T}, \quad(Q=c, b)
$$

As we discussed in the previous section, we assume that the fields include the iso-triplet SHBs with positive parity and the iso-singlet SHBs with negative parity as chiral partners to each others. They are embedded into the field $S_{Q}^{\mu}$ as

$$
S_{Q}^{\mu}=\hat{\Sigma}_{Q}^{\mu}+\hat{\Lambda}_{Q 1}^{\mu}
$$

where $\hat{\Sigma}_{Q}^{\mu}$ and $\hat{\Lambda}_{Q 1}^{\mu}$ include the iso-triplet and iso-singlet fields, respectively, as

$$
\begin{aligned}
& \hat{\Sigma}_{Q}^{\mu}=\left(\begin{array}{cc}
\Sigma_{Q}^{I=1 \mu} & \frac{1}{\sqrt{2}} \Sigma_{Q}^{I=0 \mu} \\
\frac{1}{\sqrt{2}} \Sigma_{Q}^{I=0 \mu} & \Sigma_{Q}^{I=-1 \mu}
\end{array}\right), \\
& \hat{\Lambda}_{Q 1}^{\mu}=\left(\begin{array}{cc}
0 & \frac{1}{\sqrt{2}} \Lambda_{Q 1}^{\mu} \\
-\frac{1}{\sqrt{2}} \Lambda_{Q 1}^{\mu} & 0
\end{array}\right) .
\end{aligned}
$$

These $\Sigma_{Q}^{\mu}$ and $\Lambda_{Q 1}^{\mu}$ are decomposed into spin-3/2 baryon fields and spin- $1 / 2$ fields as

$$
\begin{gathered}
\Sigma_{Q}^{\mu}=\Sigma_{Q}^{* \mu}-\frac{1}{\sqrt{3}}\left(\gamma^{\mu}+v^{\mu}\right) \gamma_{5} \Sigma_{Q}, \\
\Lambda_{Q 1}^{\mu}=\Lambda_{Q 1}^{* \mu}-\frac{1}{\sqrt{3}}\left(\gamma^{\mu}+v^{\mu}\right) \gamma_{5} \Lambda_{Q 1},
\end{gathered}
$$

where $\Sigma_{Q}^{* \mu}$ and $\Lambda_{Q 1}^{* \mu}$ denote the spin-3/2 baryon fields, and $\Sigma_{Q}$ and $\Lambda_{Q 1}$ the spin-1/2 fields, respectively. We note that the parity transformation of the $S_{Q}^{\mu}$ field is given by

$$
S_{Q}^{\mu} \stackrel{\mathrm{P}}{\rightarrow}-\gamma^{0} S_{Q \mu}^{T}
$$

where ${ }^{T}$ denotes the transposition of the $2 \times 2$ matrix in the light-quark flavor space, and that the Dirac conjugate is defined as

$$
\bar{S}_{Q}^{\mu}=S_{Q}^{\mu \dagger} \gamma^{0}
$$

We introduce a $2 \times 2$ matrix field $M$ for scalar and pseudoscalar mesons including a light quark and a light anti-quark, which belongs to the $(2,2)$ representation under the chiral $\mathrm{SU}(2)_{L} \times \mathrm{SU}(2)_{R}$ symmetry. The transformation properties of $M$ under the chiral symmetry and the parity are given by

$$
\begin{gathered}
M \stackrel{\mathrm{Ch}}{\rightarrow} g_{L} M g_{R}^{\dagger}, \\
M \stackrel{\mathrm{P}}{\rightarrow} M^{\dagger} .
\end{gathered}
$$

We assume that the effective Lagrangian terms for $M$ are constructed in such a way that the $M$ has a vacuum expectation value which breaks the chiral symmetry spontaneously, and the vacuum expectation value is proportional to the pion decay constant $f_{\pi}^{1}$ :

$$
\langle M\rangle=f_{\pi}\left(\begin{array}{ll}
1 & 0 \\
0 & 1
\end{array}\right) .
$$

In the following, for studying the decays of the single heavy baryons with emitting pions, we parametrize the field $M$ as

$$
M=f_{\pi} U,
$$

where

$$
U=e^{\frac{2 i \pi}{f_{\pi}}}
$$

with $\pi$ being the $2 \times 2$ matrix field including pions as

$$
\pi=\frac{1}{2}\left(\begin{array}{cc}
\pi^{0} & \sqrt{2} \pi^{+} \\
\sqrt{2} \pi^{-} & -\pi^{0}
\end{array}\right) .
$$

Now, let us write down an effective Lagrangian including the baryon fields $\Lambda_{Q}$ and $S_{Q}^{\mu}$ together with the meson field $M$, based on the heavy-quark spin-flavor symmetry and the chiral symmetry. We do not include the terms including more than square of $M$ field or more than two derivatives. A possible Lagrangian is given by

$$
\begin{aligned}
\mathcal{L}_{Q}= & -\operatorname{tr} \bar{S}_{Q}^{\mu}\left(v \cdot i D-\Delta_{Q}\right) S_{Q \mu}+\bar{\Lambda}_{Q}(v \cdot i D) \Lambda_{Q}+\frac{g_{1}}{2 f_{\pi}} \operatorname{tr}\left(\bar{S}_{Q}^{\mu} M^{\dagger} M S_{Q \mu}+\bar{S}_{Q \mu}^{T} M^{\dagger} S_{Q}^{\mu T}\right)-\frac{g_{2}}{2 f_{\pi}} \operatorname{tr} \bar{S}_{Q}^{\mu} M^{\dagger} S_{Q \mu}^{T} M^{T} \\
& -\frac{g_{2}^{v}}{2 m_{\Lambda_{Q}}} \operatorname{tr} \bar{S}_{Q}^{\mu} M^{\dagger} S_{Q \mu}^{T} M^{T}-i \frac{h_{1}^{I}-i h_{1}^{R}}{4 f_{\pi}^{2}} \operatorname{tr}\left(\bar{S}_{Q}^{\mu} M^{\dagger} v \cdot \partial M S_{Q \mu}+\bar{S}_{Q}^{\mu T} M v \cdot \partial M^{\dagger} S_{Q \mu}^{T}\right) \\
& -i \frac{-h_{1}^{I}-i h_{1}^{R}}{4 f_{\pi}^{2}} \operatorname{tr}\left(\bar{S}_{Q}^{\mu} v \cdot \partial M^{\dagger} M S_{Q \mu}+\bar{S}_{Q}^{\mu T} v \cdot \partial M M^{\dagger} S_{Q \mu}^{T}\right)+\frac{h_{2}}{2 f_{\pi}^{2}} \operatorname{tr}\left(\bar{S}_{Q}^{\mu} v \cdot \partial M^{\dagger} S_{Q \mu}^{T} M^{T}+\bar{S}_{Q}^{\mu T} v \cdot \partial M S_{Q \mu} M^{*}\right) \\
& -\frac{g_{3}}{2 \sqrt{2} f_{\pi}} \bar{\Lambda}_{Q} \operatorname{tr}\left(\partial^{\mu} M S_{Q \mu} \tau^{2}-\partial_{\mu} M^{\dagger} S_{Q}^{\mu T} \tau^{2}\right)+\text { H.c. },
\end{aligned}
$$

\footnotetext{
${ }^{1}$ Here we adopt the normalization of $f_{\pi}=92.4 \mathrm{MeV}$.
} 
where $m_{\Lambda_{Q}}(Q=c, b)$ are the masses of $\Lambda_{c}(2286)$ and $\Lambda_{b}$ in the ground state; $\Delta_{Q}$ provides the difference between the chiral invariant masses of $\left(\Sigma_{Q}, \Lambda_{Q 1}\right)$ chiral multiplet and the chiral singlet $\Lambda_{Q}$ with heavy-quark flavor violation included. $g_{i}(i=1,2,3), g_{2}^{v}, h_{1}^{I}, h_{1}^{R}$, and $h_{2}$ are dimensionless coupling constants. Note that we included the $g_{2}^{v}$-term to incorporate the heavy-flavor violation needed for explaining the mass differences of charm and bottom sectors. Although we can add heavy-quark flavor violation terms corresponding to the $g_{1}$-term, such contributions are absorbed into the definition of $\Delta_{Q}$. We expect that heavyquark flavor violating corrections to other terms are small.

\section{MASSES AND $\Sigma_{Q}^{(*)} \rightarrow \Lambda_{Q} \pi$ DECAYS}

In this section, we determine the coupling constants $g_{2}$ and $g_{2}^{v}$ from masses of relevant heavy baryons, and $g_{3}$ from $\Sigma_{c}^{(*)} \rightarrow \Lambda_{c} \pi$ decays. Then we make predictions of $\Sigma_{b}^{(*)} \rightarrow$ $\Lambda_{b} \pi$ decays.

When the chiral symmetry is spontaneously broken, the light meson field $M$ acquires its vacuum expectation value as in Eq. (23). Then the masses of $\Sigma_{Q}^{(*)}$ and $\Lambda_{Q 1}^{(*)}$ are expressed as

$$
\begin{aligned}
& m\left(\Sigma_{Q}^{(*)}\right)=m_{\Lambda_{Q}}+\Delta_{Q}+g_{1} f_{\pi}-\frac{g_{2}^{Q}}{2} f_{\pi}, \\
& m\left(\Lambda_{Q 1}^{(*)}\right)=m_{\Lambda_{Q}}+\Delta_{Q}+g_{1} f_{\pi}+\frac{g_{2}^{Q}}{2} f_{\pi},
\end{aligned}
$$

where $g_{2}^{Q}$ is

$$
g_{2}^{Q}=g_{2}+g_{2}^{v} \frac{f_{\pi}}{m_{\Lambda_{Q}}}
$$

In the present analysis, we assume that the heavyquark multiplet of $\left(\Lambda_{c 1}, \Lambda_{c 1}^{*}\right)=\left(\Lambda_{c}\left(2595 ; J^{P}=1 / 2^{-}\right)\right.$, $\left.\Lambda_{c}\left(2625 ; 3 / 2^{-}\right)\right)$is the chiral partner to the multiplet of $\left(\Sigma_{c}, \Sigma_{c}^{*}\right)=\left(\Sigma_{c}\left(2455 ; 1 / 2^{+}\right), \Sigma_{c}\left(2520 ; 3 / 2^{+}\right)\right)$, and that $\left(\Lambda_{b 1}, \Lambda_{b 1}^{*}\right)=\left(\Lambda_{b}\left(5912 ; 1 / 2^{-}\right), \Lambda_{b}\left(5920 ; 3 / 2^{-}\right)\right)$to $\left(\Sigma_{b}\right.$, $\left.\Sigma_{b}^{*}\right)=\left(\Sigma_{b}\left(1 / 2^{+}\right), \Sigma_{b}\left(3 / 2^{+}\right)\right)$. We list experimental data of their masses and full decay widths [16] in Table I.

We determine the values of the coupling constants $g_{2}^{Q}$ $(Q=c, b)$ from the mass differences $\Delta M_{Q}$ of chiral partners in the following way: First, we separately evaluate the mass differences of chiral partners with spin- $1 / 2$ and spin-3/2 as

$$
\begin{aligned}
\Delta M_{Q}^{(1 / 2, \exp )} & =M_{\Lambda_{Q 1}}-M_{\Sigma_{Q}}, \\
\Delta M_{Q}^{(3 / 2, \exp )} & =M_{\Lambda_{Q 1}^{*}}-M_{\Sigma_{Q}^{*}}, \\
(Q=c, b), &
\end{aligned}
$$

TABLE I. Experimental data of masses and decay widths of heavy baryons included in the present analysis.

\begin{tabular}{lccc}
\hline \hline Particle & $J^{P}$ & Mass $[\mathrm{MeV}]$ & Full width $[\mathrm{MeV}]$ \\
\hline$\Lambda_{c}$ & $1 / 2^{+}$ & $2286.46 \pm 0.14$ & no strong decays \\
$\Sigma_{c}^{++}(2455)$ & $1 / 2^{+}$ & $2453.97 \pm 0.14$ & $1.89_{-0.18}^{+0.09}$ \\
$\Sigma_{c}^{+}(2455)$ & $1 / 2^{+}$ & $2452.9 \pm 0.4$ & $<4.6$ \\
$\Sigma_{c}^{0}(2455)$ & $1 / 2^{+}$ & $2453.75 \pm 0.14$ & $1.83_{-0.19}^{+0.11}$ \\
$\Sigma_{c}^{++}(2520)$ & $3 / 2^{+}$ & $2518.41_{-0.19}^{+0.21}$ & $14.78_{-0.40}^{+0.30}$ \\
$\Sigma_{c}^{+}(2520)$ & $3 / 2^{+}$ & $2517.5 \pm 1.3$ & $<17$ \\
$\Sigma_{c}^{0}(2520)$ & $3 / 2^{+}$ & $2518.48 \pm 0.20$ & $15.3_{-0.5}^{+0.4}$ \\
$\Lambda_{c}(2595)$ & $1 / 2^{-}$ & $2595.25 \pm 0.28$ & $2.59 \pm 0.30 \pm 0.47$ \\
$\Lambda_{c}(2625)$ & $3 / 2^{-}$ & $2628.11 \pm 0.19$ & $<0.97$ \\
$\Lambda_{b}$ & $1 / 2^{+}$ & $5619.58 \pm 0.17$ & no \\
$\Sigma_{b}^{+}$ & $1 / 2^{+}$ & $5811.3_{-0.8}^{+0.9} \pm 1.7$ & $9.7_{-2.8}^{+3.8+1.2}$ \\
$\Sigma_{b}^{0}$ & $1 / 2^{+}$ & $\cdots$ & $\cdots$ \\
$\Sigma_{b}^{-}$ & $1 / 2^{+}$ & $5815.5_{-0.5}^{+0.6} \pm 1.7$ & $4.9_{-2.1}^{+3.1} \pm 1.1$ \\
$\Sigma_{b}^{*+}$ & $3 / 2^{+}$ & $5832.1 \pm 0.7_{-1.8}^{+1.7}$ & $11.5_{-2.2}^{+2.7}+1.0$ \\
$\Sigma_{b}^{* 0}$ & $3 / 2^{+}$ & $\cdots$ & $\cdots$ \\
$\Sigma_{b}^{*-}$ & $3 / 2^{+}$ & $5835.1 \pm 0.6_{-1.8}^{+1.7}$ & $7.5_{-1.8}^{+2.2}+0.9$ \\
$\Lambda_{b}(5912)$ & $1 / 2^{-}$ & $5912.18 \pm 0.13 \pm 0.17$ & $<0.66$ \\
$\Lambda_{b}(5920)$ & $3 / 2^{-}$ & $5919.90 \pm 0.19$ & $<0.63$ \\
\hline \hline & & &
\end{tabular}

where $M_{\Lambda_{Q 1}^{(*)}}$ and $M_{\Sigma_{Q}^{(*)}}$ are given by taking the isospin average of relevant masses. Using the values listed in Table I, we obtain

$$
\begin{aligned}
& \frac{\Delta M_{c}^{(1 / 2, \exp )}}{f_{\pi}}=1.19, \\
& \frac{\Delta M_{c}^{(3 / 2, \exp )}}{f_{\pi}}=1.53
\end{aligned}
$$

for the charm sector. By taking the spin average of these values, we determine the center value of $g_{2}^{c}$ as

$$
g_{2}^{c}=\frac{1}{3}\left(\frac{\Delta M_{c}^{(1 / 2, \exp )}}{f_{\pi}}+2 \frac{\Delta M_{c}^{(3 / 2, \exp )}}{f_{\pi}}\right)=1.30 .
$$

By taking the violation of heavy-spin symmetry, we evaluate the error as

$$
g_{2}^{c}=1.30_{-|1.19-1.30|}^{+|1.53-1.30|}=1.30_{-0.11}^{+0.23} .
$$

Similarly, $g_{2}^{b}$ is evaluated as

$$
g_{2}^{b}=0.980_{-0.046}^{+0.090}
$$

Let us determine the value of the coupling constant $g_{3}$ from $\Sigma_{c}^{(*)} \rightarrow \Lambda_{c} \pi$ decays. We use the experimental values 
of the full widths of $\Sigma_{c}^{++}\left(2455 ; 1 / 2^{+}\right), \Sigma_{c}^{0}\left(2455 ; 1 / 2^{+}\right)$, $\Sigma_{c}^{*++}\left(2520 ; 3 / 2^{+}\right)$, and $\Sigma_{c}^{* 0}\left(2520 ; 3 / 2^{+}\right)$with assuming that the one-pion decay is the dominant decay mode for each particle. We first calculate four values of the effective couplings $g_{3}\left(\Sigma_{c}^{(*)++} \rightarrow \Lambda_{c}^{(*)+} \pi^{+}\right)$and $g_{3}\left(\Sigma_{c}^{(*) 0} \rightarrow \Lambda_{c}^{(*)+} \pi^{-}\right)$ from the corresponding decay widths. Then, taking the isospin average for $J^{P}=1 / 2^{+}$and $3 / 2^{+}$separately, we obtain

$$
\begin{aligned}
& g_{3}^{(1 / 2)}=\frac{g_{3}\left(\Sigma_{c}^{++} \rightarrow \Lambda_{c}^{+} \pi^{+}\right)+g_{3}\left(\Sigma_{c}^{0} \rightarrow \Lambda_{c}^{+} \pi^{-}\right)}{2}=0.673, \\
& g_{3}^{(3 / 2)}=\frac{g_{3}\left(\Sigma_{c}^{*++} \rightarrow \Lambda_{c}^{+} \pi^{+}\right)+g_{3}\left(\Sigma_{c}^{* 0} \rightarrow \Lambda_{c}^{+} \pi^{-}\right)}{2}=0.695 .
\end{aligned}
$$

The spin average of the above values are calculated as

$$
g_{3}=\frac{1}{3}\left(g_{3}^{1 / 2}+2 g_{3}^{3 / 2}\right)=0.688 .
$$

We include the systematic error of the spin average and the statistical error of the experimental data as

$$
g_{3}+\left|g_{3}^{3 / 2}-g_{3}\right|+\text { stat.e. }
$$

to obtain

$$
g_{3}=0.688_{-0.025}^{+0.013} \text {. }
$$

We summarize the estimated values of $g_{2}^{Q}$ and $g_{3}$ in Table II. Using the estimated value of $g_{3}$, we calculate the decay widths of $\Sigma_{Q}^{(*)} \rightarrow \Lambda_{Q} \pi$ as shown in Table III. This shows that the obtained widths of $\Sigma_{c}\left(J^{P}=1 / 2^{+}\right)$and $\Sigma_{c}^{*}\left(3 / 2^{+}\right)$are consistent with each other even though we used the common coupling constant $g_{3}$. This implies that heavy-quark spin violation between them is small. Furthermore, the predicted widths of $\Sigma_{b}$ and $\Sigma_{b}^{*}$ obtained by the common $g_{3}$ coupling for charm and bottom sectors are consistent with experiments. This indicates that the violation of the heavy-quark flavor symmetry is small at this moment, but precise determination of them by future experiments might require the inclusion of heavy-quark flavor violation. We should note that the predicted widths of $\Sigma_{c}^{(*)+} \rightarrow \Lambda_{c}^{+} \pi^{0}$ are larger than those of their isospin partners since the phase space is larger due to the smallness of the mass of $\pi^{0}$.

TABLE II. Estimated values of the coupling constants $g_{2}^{Q}$ and $g_{3}$.

\begin{tabular}{lc}
\hline \hline Parameter & Value \\
\hline$g_{2}^{c}$ & $1.30_{-0.11}^{+0.23}$ \\
$g_{2}^{b}$ & $0.980_{-0.046}^{+0.090}$ \\
$g_{3}$ & $0.688_{-0.025}^{+0.013}$ \\
\hline \hline
\end{tabular}

TABLE III. Decay widths $\Sigma_{Q}^{(*)} \rightarrow \Lambda_{Q} \pi$ calculated in our model. We use $\Sigma_{c}^{++} \rightarrow \Lambda_{c}^{+} \pi^{+}, \Sigma_{c}^{0} \rightarrow \Lambda_{c}^{+} \pi^{-}, \Sigma_{c}^{*++} \rightarrow \Lambda_{c}^{+} \pi^{+}$, and $\Sigma_{c}^{* 0} \rightarrow$ $\Lambda_{c}^{+} \pi^{-}$to determine the coupling constant $g_{3}$ as explained in the text.

Decay modes Our model [MeV] Experimental values [MeV]

\begin{tabular}{lcc}
\hline$\Sigma_{c}^{++} \rightarrow \Lambda_{c}^{+} \pi^{+}$ & $1.96_{-0.14}^{+0.07}$ & $1.89_{-0.18}^{+0.09}$ \\
$\Sigma_{c}^{+} \rightarrow \Lambda_{c}^{+} \pi^{0}$ & $2.28_{-0.17}^{+0.09}$ & $<4.6$ \\
$\Sigma_{c}^{0} \rightarrow \Lambda_{c}^{+} \pi^{-}$ & $1.94_{-0.14}^{+0.07}$ & $1.83_{-0.19}^{+0.11}$ \\
$\Sigma_{c}^{*++} \rightarrow \Lambda_{c}^{+} \pi^{+}$ & $14.7_{-1.1}^{+0.6}$ & $14.78_{-0.40}^{+0.30}$ \\
$\Sigma_{c}^{*+} \rightarrow \Lambda_{c}^{+} \pi^{0}$ & $15.3_{-1.1}^{+0.6}$ & $<17$ \\
$\Sigma_{c}^{* 0} \rightarrow \Lambda_{c}^{-} \pi^{0}$ & $14.7_{-1.1}^{+0.6}$ & $15.3_{-0.5}^{+0.4}$ \\
$\Sigma_{b}^{+} \rightarrow \Lambda_{b}^{0} \pi^{+}$ & $6.14_{-0.45}^{+0.23}$ & $9.7_{-2.8}^{+3.8+1.2}$ \\
$\Sigma_{b}^{0} \rightarrow \Lambda_{b}^{0} \pi^{0}$ & $7.27_{-0.53}^{+0.27}$ & $\cdots$ \\
$\Sigma_{b}^{-} \rightarrow \Lambda_{b}^{0} \pi^{-}$ & $7.02_{-0.51}^{+0.27}$ & $4.9_{-2.1}^{+3.1} \pm 1.1$ \\
$\Sigma_{b}^{*+} \rightarrow \Lambda_{b}^{0} \pi^{+}$ & $11.0_{-0.8}^{+0.4}$ & $11.5_{-2.2}^{+2.7}{ }_{-1.5}^{+1.0}$ \\
$\Sigma_{b}^{* 0} \rightarrow \Lambda_{b}^{0} \pi^{0}$ & $12.3_{-0.9}^{+0.5}$ & $\cdots$ \\
$\Sigma_{b}^{*-} \rightarrow \Lambda_{b}^{0} \pi^{-}$ & $11.9_{-0.9}^{+0.4}$ & $7.5_{-1.8}^{+2.2}+0.9$ \\
\hline \hline
\end{tabular}

\section{V. $\Lambda_{Q 1}^{(*)} \rightarrow \Lambda_{Q} \pi \pi$ DECAYS}

In this section, we consider $\Lambda_{Q 1}^{(*)} \rightarrow \Lambda_{Q} \pi \pi$ decays. In Fig. 1, we plot the relevant diagrams for $\Lambda_{Q 1}^{(*)} \rightarrow \Lambda_{Q} \pi^{+} \pi^{-}$in our model. In the diagrams (a), (b), and (d), $\Sigma_{Q}^{(*)}$ s appear as intermediate states, while in diagrams (c) and (e), $\Lambda_{Q 1}^{(*)}$ and

(a) $\Lambda$

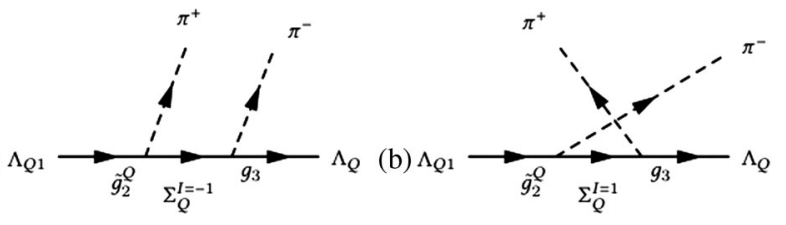

(c) $\Lambda_{Q 1}$

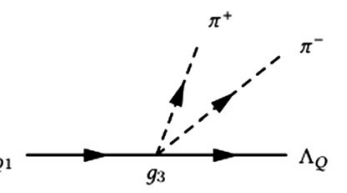

(d) $\Lambda_{Q 1}$

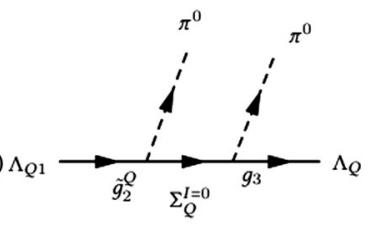

(e) $\Lambda_{Q 1}$

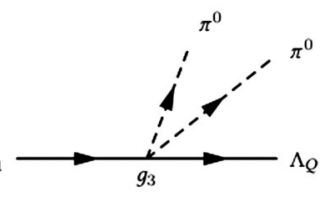

FIG. 1. Feynman diagrams contributing to $\Lambda_{Q 1} \rightarrow \Lambda_{Q} \pi \pi$ decay. (a) $\Sigma_{Q}$ with the isospin $I=-1$ appears in the intermediate state; (b) $\Sigma_{Q}$ with $I=1$ appears; (c) the non-resonant contribution to $\pi^{+} \pi^{-}$mode; (d) $\Sigma_{Q}$ with $I=0$ appears; (e) the nonresonant contribution to $\pi^{0} \pi^{0}$ mode. The effective coupling $\tilde{g}_{2}^{Q}$ is defined as $\tilde{g}_{2}^{Q}=g_{2}^{Q}+\left(h_{1}^{I}+i h_{2}\right) \frac{E_{\pi}}{f_{\pi}}$, where $E_{\pi}$ is the relevant pion energy. Similar Feynman diagrams contribute to $\Lambda_{Q 1}^{*} \rightarrow \Lambda_{Q} \pi \pi$ decay. 
$\Lambda_{Q}$ couple to two pions directly. It should be noticed that, due to the chiral partner structure, the coupling constant in (c) and (e) is equivalent to the $\Sigma_{Q}^{(*)} \rightarrow \Lambda_{Q} \pi$ coupling in (a), (b), and (d). Then, it is not suitable to drop the contributions in (c) and (e). Actually, as we will show below, They are not negligible for $\Lambda_{c 1}^{*}$ and $\Lambda_{b 1}^{(*)}$ decays.

From the diagrams in Fig. 1, the amplitude of $\Lambda_{Q 1} \rightarrow$ $\Lambda_{Q} \pi^{+} \pi^{-}$decays is calculated as

$$
\begin{aligned}
\mathcal{M}= & -\frac{g_{3}}{\sqrt{3} f_{\pi}^{2}}\left(p_{2}^{\mu}+p_{3}^{\mu}\right) \bar{u}\left(p_{1}, t\right)\left(\gamma_{\mu}+\frac{P_{\mu}}{M}\right) \gamma_{5} u_{1}(P, s) \\
& -\frac{g_{3}}{\sqrt{3} f_{\pi}}\left\{g_{2}+\left(h_{1}^{I}+i h_{2}\right) \frac{E_{2}\left(p_{2}\right)}{f_{\pi}}\right\} S_{f}^{++}(q) p_{3}^{\mu} \bar{u}\left(p_{1}, t\right)\left(\gamma_{\mu}+\frac{q_{\mu}}{m^{++}}\right) \gamma_{5}\left(m^{++}+\not 1\right) u_{1}(P, s) \\
& -\frac{g_{3}}{\sqrt{3} f_{\pi}}\left\{g_{2}+\left(h_{1}^{I}+i h_{2}\right) \frac{E_{3}\left(p_{3}\right)}{f_{\pi}}\right\} S_{f}^{0}(k) p_{2}^{\mu} \bar{u}\left(p_{1}, t\right)\left(\gamma_{\mu}+\frac{k_{\mu}}{m^{0}}\right) \gamma_{5}\left(m^{0}+\not k\right) u_{1}(P, s),
\end{aligned}
$$

where $P$ is the initial momentum of $\Lambda_{Q 1}, p_{1}$ the momentum of $\Lambda_{Q}, p_{2}$ and $p_{3}$ are the momenta of pions, and $k$ and $q$ the momenta of intermediate $\Sigma_{Q} \mathrm{~s} . S_{f}$ is the propagator for the intermediate $\Sigma_{Q}$ s given by

$$
S_{f}(k) \equiv \frac{1}{m_{\Sigma_{Q}}^{2}-k^{2}+i m_{\Sigma_{Q}} \Gamma_{\Sigma_{Q}}},
$$

where $m_{\Sigma_{Q}}$ and $\Gamma_{\Sigma_{Q}}$ are the mass and decay width of intermediate $\Sigma_{Q}$. We used isospin-averaged values of masses and decay widths in the present analysis. Similarly, the amplitude $\Lambda_{Q 1} \rightarrow \Lambda_{Q} \pi^{0} \pi^{0}$ decays is

$$
\begin{aligned}
\mathcal{M}= & -\frac{g_{3}}{\sqrt{3} f_{\pi}^{2}}\left(p_{2}^{\mu}+p_{3}^{\mu}\right) \bar{u}\left(p_{1}, t\right)\left(\gamma_{\mu}+\frac{P_{\mu}}{M}\right) \gamma_{5} u_{1}(P, s) \\
& -\frac{g_{3}}{\sqrt{3} f_{\pi}}\left\{g_{2}+\left(h_{1}^{I}+i h_{2}\right) \frac{E_{3}\left(p_{3}\right)}{f_{\pi}}\right\} S_{f}^{+}(k) p_{2}^{\mu} \bar{u}\left(p_{1}, t\right)\left(\gamma_{\mu}+\frac{k_{\mu}}{m^{+}}\right) \gamma_{5}\left(m^{+}+\not k\right) u_{1}(P, s) .
\end{aligned}
$$

We determine the relation between the values of $h_{1}^{I}$ and $h_{2}$ from the full width of $\Lambda_{c 1}\left(\Lambda_{c}(2595)\right)$. Taking into account the errors of $g_{2}^{c}, g_{3}$ and the total width with $\Lambda_{c 1}$, we determine the allowed range of $h_{1}^{I}$ and $h_{2}$ as shown in Fig. 2. Using these values we calculate the two-pion decay widths of $\Lambda_{c}(2625), \Lambda_{b}(5912)$, and $\Lambda_{b}(5920)$, which are

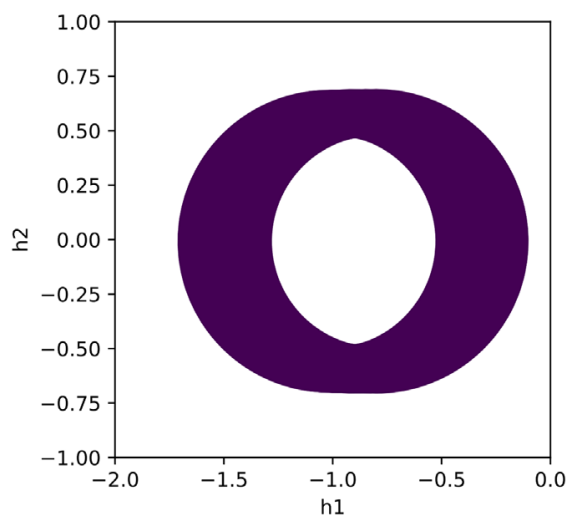

FIG. 2. Allowed range of $h_{1}^{I}$ and $h_{2}$ shown by purple area. summarized in Table IV. The predicted decay width of $\Lambda_{c}(2625)$ is consistent with predictions of a quark model in Refs. $[12,13]$. We note that the predicted decay widths of $\Lambda_{b}(5912)$ and $\Lambda_{b}(5920)$ are extremely tiny due to the phase

TABLE IV. Predicted widths of $\Lambda_{Q 1} \rightarrow \Lambda_{Q} \pi \pi$ decays.

\begin{tabular}{lccc}
\hline \hline Initial & Mode & $\begin{array}{c}\text { Our model } \\
{[\mathrm{MeV}]}\end{array}$ & $\begin{array}{c}\text { Experimental } \\
\text { values [MeV] }\end{array}$ \\
\hline$\Lambda_{c}(2595)$ & $\Lambda_{c} \pi^{+} \pi^{-}$ & $0.562-1.09$ & \\
& $\Lambda_{c} \pi^{0} \pi^{0}$ & $1.23-2.31$ & \\
& sum & $1.82-3.36$ (input) & $2.59 \pm 0.30 \pm 0.47$ \\
$\Lambda_{c}(2625)$ & $\Lambda_{c} \pi^{+} \pi^{-}$ & $0.0618-0.507$ & \\
& $\Lambda_{c} \pi^{0} \pi^{0}$ & $0.0431-0.226$ & \\
& sum & $0.106-0.733$ & $<0.97$ \\
$\Lambda_{b}(5912)$ & $\Lambda_{b} \pi^{+} \pi^{-}$ & $(0.67-4.4) \times 10^{-3}$ & \\
& $\Lambda_{b} \pi^{0} \pi^{0}$ & $(1.4-6.0) \times 10^{-3}$ & \\
& sum & $(2.1-10) \times 10^{-3}$ & $<0.66$ \\
$\Lambda_{b}(5920)$ & $\Lambda_{b} \pi^{+} \pi^{-}$ & $(0.75-13) \times 10^{-3}$ & \\
& $\Lambda_{b} \pi^{0} \pi^{0}$ & $(2.2-12) \times 10^{-3}$ & \\
& sum & $(3.0-25) \times 10^{-3}$ & $<0.63$ \\
\hline \hline
\end{tabular}


TABLE V. Four typical parameter sets determined from $\Lambda_{c 1} \rightarrow$ $\Lambda_{c} \pi \pi$ decay width.

\begin{tabular}{lccclc}
\hline \hline Set & $g_{2}^{c}$ & $g_{2}^{b}$ & $g_{3}$ & \multicolumn{1}{c}{$h_{1}^{I}$} & \multicolumn{1}{c}{$h_{2}$} \\
\hline Set 1 & 1.30 & 0.980 & 0.688 & -0.277 & 0 \\
Set 2 & 1.30 & 0.980 & 0.688 & -1.45 & 0 \\
Set 3 & 1.30 & 0.980 & 0.688 & -0.450 & 0.500 \\
Set 4 & 1.30 & 0.980 & 0.688 & -1.00 & -0.500 \\
\hline \hline
\end{tabular}

space suppression. As we will show in the next section, the radiative decay widths for $\Lambda_{b}(5912)$ and $\Lambda_{b}(5920)$ are comparable with or even larger than the hadronic decay widths.

Here we pick up several typical choices of $h_{1}^{I}$ and $h_{2}$, and study the contributions of the diagrams in Fig. 1 and their interferences. In Table V, we list four typical sets of $h_{1}^{I}$ and $h_{2}$ together with the values of $g_{2}^{Q}$ and $g_{3}$.
Using four sets of parameters in Table $\mathrm{V}$, we study contributions of intermediate states to $\Lambda_{Q 1}^{(*)} \rightarrow \Lambda_{Q} \pi \pi$ decays, which are shown in Tables VI-IX. Table VI shows the contributions of intermediate states to $\Lambda_{c}\left(2595 ; 1 / 2^{-}\right) \rightarrow$ $\Lambda_{c} \pi \pi$ decay. More than half of this decay width is provided by the contribution in which $\Sigma_{c}^{+}$exists as an intermediate state as in Fig. 1(d). This is because the threshold for $\Lambda_{c}(2595) \rightarrow \Sigma_{c}^{+}(2455) \pi^{0}$ decay is open. Accordingly, nonresonant (NR) contributions in Figs. 1(c) and 1(e) are very small. On the other hand, NR contributions to $\Lambda_{c}(2625)$, $\Lambda_{b}(5912)$, and $\Lambda_{b}(5920)$ are comparable to resonant contributions, partly because the threshold for $\Sigma_{Q} \pi$ decays are not open. As we stressed, the coupling constant $g_{3}$ in Figs. 1(c) and 1(e) is fixed from $\Sigma_{c} \rightarrow \Lambda_{c} \pi$ decay based on the chiral partner structure. Then, the experimental check of nonresonant contributions will give a clue to the chiral symmetry structure.

TABLE VI. Contributions of intermediate states to $\Lambda_{c}\left(2595 ; 1 / 2^{-}\right) \rightarrow \Lambda_{c} \pi \pi$ decay. "(c-NR)" and "(e-NR)" in the column for "intermediate states" indicate the nonresonant contributions expressed in Figs. 1(c) and 1(e), respectively. "(a- $\left.\Sigma_{c}^{0}\right)$ ", "(b- $\left.\Sigma_{c}^{++}\right)$," and " $\left(\mathrm{d}-\Sigma_{c}^{+}\right)$" indicate the resonant contributions in Figs. 1(a), 1(b), and 1(d), respectively. "(b- $\left.\Sigma_{c}^{++}\right)$and $\left(\mathrm{a}-\Sigma_{c}^{0}\right)$ " and so on indicate the contributions of the interferences.

\begin{tabular}{lccccc}
\hline \hline Decay mode & Intermediate states & Set $1[\mathrm{keV}]$ & Set 2 [keV] & Set 3 [keV] & Set 4 [keV] \\
\hline$\Lambda_{c}\left(2595 ; 1 / 2^{-}\right) \rightarrow \Lambda_{c}^{+} \pi^{+} \pi^{-}$ & $(\mathrm{c}-\mathrm{NR})$ & 4.10 & 4.10 & 4.10 & 4.10 \\
& $\left(\mathrm{~b}-\Sigma_{c}^{++}\right)$ & 344 & 408 & 438 & 302 \\
& $\left(\mathrm{a}-\Sigma_{c}^{0}\right)$ & 390 & 466 & 497 & 344 \\
& $\left(\mathrm{~b}-\Sigma_{c}^{++}\right)$and $\left(\mathrm{a}-\Sigma_{c}^{0}\right)$ & 15.7 & 26.4 & 21.5 & 18.2 \\
& $(\mathrm{c}-\mathrm{NR})$ and $\left(\mathrm{b}-\Sigma_{c}^{++}\right)$ & 42.7 & -49.1 & 36.3 & -21.1 \\
& $(\mathrm{c}-\mathrm{NR})$ and $\left(\mathrm{a}-\Sigma_{c}^{0}\right)$ & 44.3 & -51.5 & 38.1 & -22.6 \\
$\Lambda_{c}\left(2595 ; 1 / 2^{-}\right) \rightarrow \Lambda_{c}^{+} \pi^{0} \pi^{0}$ & $(\mathrm{e}-\mathrm{NR})$ & 4.85 & 4.85 & 4.85 & 4.85 \\
& $\left(\mathrm{~d}-\Sigma_{c}^{+}\right)$ & $1.71 \times 10^{3}$ & $1.82 \times 10^{3}$ & $2.12 \times 10^{3}$ & $1.39 \times 10^{3}$ \\
Total & $(\mathrm{e}-\mathrm{NR})$ and $\left(\mathrm{d}-\Sigma_{c}^{+}\right)$ & 33.1 & -41.6 & 63.7 & -54.6 \\
\hline \hline
\end{tabular}

TABLE VII. Contributions of intermediate states to $\Lambda_{c}\left(2625 ; 3 / 2^{-}\right) \rightarrow \Lambda_{c} \pi \pi$ decay. "(c-NR)" and "(e-NR)" in the column for "intermediate states" indicate the nonresonant contributions expressed in Figs. 1(c) and 1(e), respectively. " $\left(\mathrm{a}-\Sigma_{c}^{* 0}\right)$ ", " $\left(\mathrm{b}-\Sigma_{c}^{*++}\right)$, , and " $\left(\mathrm{d}-\Sigma_{c}^{*+}\right)$ " indicate the resonant contributions in Figs. 1(a), 1(b), and $1(\mathrm{~d})$, respectively. " $\left(\mathrm{b}-\Sigma_{c}^{*++}\right)$ and $\left(\mathrm{a}-\Sigma_{c}^{* 0}\right)$ " and so on indicate the contributions of the interferences.

\begin{tabular}{lccccc}
\hline \hline Decay mode & Intermediate states & Set $1[\mathrm{keV}]$ & Set $2[\mathrm{keV}]$ & Set 3 [keV] & Set $4[\mathrm{keV}]$ \\
\hline$\Lambda_{c}\left(2625 ; 3 / 2^{-}\right) \rightarrow \Lambda_{c}^{+} \pi^{+} \pi^{-}$ & $(\mathrm{c}-\mathrm{NR})$ & 58.4 & 58.4 & 58.4 & 58.4 \\
& $\left(\mathrm{~b}-\Sigma_{c}^{*++}\right)$ & 78.2 & 149 & 113 & 97.8 \\
& $\left(\mathrm{a}-\Sigma_{c}^{* 0}\right)$ & 76.7 & 157 & 114 & 102 \\
& $\left(\mathrm{~b}-\Sigma_{c}^{*++}\right)$ and $\left(\mathrm{a}-\Sigma_{c}^{* 0}\right)$ & 7.44 & 22.0 & 12.5 & 13.6 \\
& $(\mathrm{c}-\mathrm{NR})$ and $\left(\mathrm{b}-\Sigma_{c}^{*++}\right)$ & 93.2 & -135 & 74.8 & -62.8 \\
& $(\mathrm{c}-\mathrm{NR})$ and $\left(\mathrm{a}-\Sigma_{c}^{* 0}\right)$ & 92.3 & -138 & 74.3 & -65.3 \\
$\Lambda_{c}\left(2625 ; 3 / 2^{-}\right) \rightarrow \Lambda_{c}^{+} \pi^{0} \pi^{0}$ & $(\mathrm{e}-\mathrm{NR})$ & 43.0 & 43.0 & 43.0 & 43.0 \\
& $\left(\mathrm{~d}-\Sigma_{c}^{*+}\right)$ & 69.1 & 125 & 97.8 & 83.1 \\
Total & $(\mathrm{e}-\mathrm{NR})$ and $\left(\mathrm{d}-\Sigma_{c}^{*+}\right)$ & 73.2 & -105 & 60.4 & -50.1 \\
\hline \hline
\end{tabular}


TABLE VIII. Contributions of intermediate states to $\Lambda_{b}\left(5912 ; 1 / 2^{-}\right) \rightarrow \Lambda_{b} \pi \pi$ decay. "(c-NR)" and "(e-NR)" in the column for "intermediate states" indicate the nonresonant contributions expressed in Figs. 1(c) and 1(e), respectively. " $\left(\mathrm{a}-\Sigma_{b}^{-}\right)$, " "(b- $\left.\Sigma_{b}^{+}\right)$, , and " $\left(\mathrm{d}-\Sigma_{b}^{0}\right)$ " indicate the resonant contributions in Figs. 1(a), 1(b), and 1(d), respectively. " $\left(\mathrm{b}-\Sigma_{b}^{+}\right)$and $\left(\mathrm{a}-\Sigma_{b}^{-}\right)$" and so on indicate the contributions of the interferences.

\begin{tabular}{lccccc}
\hline \hline Decay mode & Intermediate states & Set 1 [keV] & Set 2 [keV] & Set 3 [keV] & Set 4 [keV] \\
\hline$\Lambda_{b}\left(5912 ; 1 / 2^{-}\right) \rightarrow \Lambda_{b}^{0} \pi^{+} \pi^{-}$ & $(\mathrm{c}-\mathrm{NR})$ & 0.61 & 0.61 & 0.61 & 0.61 \\
& $\left(\mathrm{~b}-\Sigma_{b}^{+}\right)$ & 0.42 & 2.3 & 0.95 & 1.3 \\
& $\left(\mathrm{a}-\Sigma_{b}^{-}\right)$ & 0.35 & 1.9 & 0.80 & 1.1 \\
& $\left(\mathrm{~b}-\Sigma_{b}^{+}\right)$and $\left(\mathrm{a}-\Sigma_{b}^{-}\right)$ & 0.018 & 0.11 & 0.043 & 0.062 \\
& $(\mathrm{c}-\mathrm{NR})$ and $\left(\mathrm{b}-\Sigma_{b}^{+}\right)$ & 0.71 & -1.7 & 0.47 & -0.87 \\
& $(\mathrm{c}-\mathrm{NR})$ and $\left(\mathrm{a}-\Sigma_{b}^{-}\right)$ & 0.65 & -1.5 & 0.38 & -0.75 \\
$\Lambda_{b}\left(5912 ; 1 / 2^{-}\right) \rightarrow \Lambda_{b}^{0} \pi^{0} \pi^{0}$ & $(\mathrm{e}-\mathrm{NR})$ & 1.40 & 1.40 & 1.40 & 1.40 \\
& $\left(\mathrm{~d}-\Sigma_{b}^{0}\right)$ & 0.97 & 5.0 & 2.1 & 2.9 \\
Total & $(\mathrm{e}-\mathrm{NR})$ and $\left(\mathrm{d}-\Sigma_{b}^{0}\right)$ & 1.6 & -3.8 & 1.0 & -1.9 \\
\hline \hline
\end{tabular}

\section{RADIATIVE DECAYS}

In this section, we consider radiative decays of the heavy baryons. The relevant Lagrangian is given by

$$
\begin{aligned}
\mathcal{L}_{\text {rad }}= & \frac{r_{1}}{F} \operatorname{tr}\left(\bar{S}_{Q}^{\mu} Q_{\text {light }} S_{Q}^{\nu}+\bar{S}_{Q}^{\mu T} Q_{\text {light }} S_{Q}^{\nu T}\right) F_{\mu \nu} \\
& +\frac{r_{2}}{F} \operatorname{tr}\left(\bar{S}_{Q}^{\mu} Q_{\text {light }} S_{Q}^{\nu}-\bar{S}_{Q}^{\mu T} Q_{\text {light }} S_{Q}^{\nu T}\right) \tilde{F}_{\mu \nu} \\
& +\frac{r_{3}}{F^{2}} \bar{\Lambda}_{Q} \operatorname{tr}\left(S_{Q}^{\mu} \tau^{2} M Q_{\text {light }} v^{\nu}-S_{Q}^{\mu T} \tau^{2} M^{\dagger} Q_{\text {light }} v^{\nu}\right) F_{\mu \nu} \\
& + \text { H.c. }+\frac{r_{4}}{F^{2}} \bar{\Lambda}_{Q} \operatorname{tr}\left(S_{Q}^{\mu} \tau^{2} M Q_{\text {light }} v^{\nu}\right. \\
& \left.+S_{Q}^{\mu T} \tau^{2} M^{\dagger} Q_{\text {light }} v^{\nu}\right) \tilde{F}_{\mu \nu}+\text { H.c. },
\end{aligned}
$$

where $F_{\mu \nu}$ is the field strength of the photon and $\tilde{F}_{\mu \nu}$ is its dual tensor: $\tilde{F}_{\mu \nu}=(1 / 2) \epsilon_{\mu \nu \rho \sigma} F^{\rho \sigma} \cdot r_{i}(i=1, \ldots, 4)$ are dimensionless constants, and $F$ is a constant with dimension one. In this analysis, we take $F=350 \mathrm{MeV}$ following Ref. [14]. We note that the values of the constants $r_{i}$ are of order one based on quark models [14].

Let us first study the electromagnetic intramultiplet transitions governed by the $r_{1}$-term in Eq. (44). Let $B^{*}$ denotes the decaying baryon with spin-3/2 $\left(B^{*}=\Lambda_{Q 1}^{*}\right.$, $\left.\Sigma_{Q}^{*}\right)$, and $B$ the daughter baryon with spin- $1 / 2\left(B=\Lambda_{Q 1}\right.$, $\left.\Sigma_{Q}\right)$. Then the radiative decay width is given by

$$
\Gamma_{B^{*} \rightarrow B \gamma}=C_{B^{*} B \gamma}^{2} \frac{16 \alpha r_{1}^{2}}{9 F^{2}} \frac{m_{B}}{m_{B^{*}}} E_{\gamma}^{3}
$$

where $\alpha$ is the electromagnetic fine structure constant, $E_{\gamma}$ is the photon energy, and $C_{B^{*} B \gamma}$ is the Clebsh-Gordon constant given by

TABLE IX. Contributions of intermediate states to $\Lambda_{b}\left(5920 ; 3 / 2^{-}\right) \rightarrow \Lambda_{c} \pi \pi$ decay. "(c-NR)" and "(e-NR)" in the column for "intermediate states" indicate the nonresonant contributions expressed in Figs. 1(c) and 1(e), respectively. "(a- $\left.\Sigma_{b}^{*-}\right)$ ", "(b- $\left.\Sigma_{b}^{*+}\right)$ " and "(d- $\left.\Sigma_{b}^{* 0}\right)$ " indicate the resonant contributions in Figs. 1(a), 1(b) and 1(d), respectively. " $\left(\mathrm{b}-\Sigma_{b}^{*+}\right)$ and $\left(\mathrm{a}-\Sigma_{b}^{*-}\right)$ " and so on indicate the contributions of the interferences.

\begin{tabular}{lccccc}
\hline \hline Decay mode & Intermediate states & Set $1[\mathrm{keV}]$ & Set $2[\mathrm{keV}]$ & Set 3 $[\mathrm{keV}]$ & Set $4[\mathrm{keV}]$ \\
\hline$\Lambda_{b}\left(5920 ; 3 / 2^{-}\right) \rightarrow \Lambda_{b}^{0} \pi^{+} \pi^{-}$ & $(\mathrm{c}-\mathrm{NR})$ & 2.4 & 2.4 & 2.4 & 2.4 \\
& $\left(\mathrm{~b}-\Sigma_{b}^{*+}\right)$ & 0.87 & 5.2 & 2.1 & 3.0 \\
& $\left(\mathrm{a}-\Sigma_{b}^{*-}\right)$ & 0.80 & 4.8 & 1.9 & 2.8 \\
& $\left(\mathrm{~b}-\Sigma_{b}^{*+}\right)$ and $\left(\mathrm{a}-\Sigma_{b}^{*-}\right)$ & 0.040 & 0.27 & 0.10 & 0.15 \\
& $(\mathrm{c}-\mathrm{NR})$ and $\left(\mathrm{b}-\Sigma_{b}^{*+}\right)$ & 2.1 & -5.1 & 1.3 & -2.6 \\
& $(\mathrm{c}-\mathrm{NR})$ and $\left(\mathrm{a}-\Sigma_{b}^{*-}\right)$ & 2.0 & -4.9 & 1.1 & -2.4 \\
$\Lambda_{b}\left(5920 ; 3 / 2^{-}\right) \rightarrow \Lambda_{b}^{0} \pi^{0} \pi^{0}$ & $(\mathrm{e}-\mathrm{NR})$ & 3.5 & 3.5 & 3.5 & 3.5 \\
& $\left(\mathrm{~d}-\Sigma_{b}^{* 0}\right)$ & 1.3 & 7.3 & 3.0 & 4.2 \\
Total & $(\mathrm{e}-\mathrm{NR})$ and $\left(\mathrm{d}-\Sigma_{b}^{* 0}\right)$ & 3.0 & -7.2 & 1.9 & -3.7 \\
\hline \hline
\end{tabular}


TABLE X. Radiative decay widths of $\Lambda_{Q 1}^{*} \rightarrow \Lambda_{Q 1} \gamma$ and $\Sigma_{Q}^{*} \rightarrow$ $\Sigma_{Q} \gamma$ in units of $\mathrm{keV}$. The values in the row indicated by "Predictions" are our predicted values, where $r_{1}$ is an undetermined parameter of $\mathcal{O}(1)$. For comparison, we list predictions in Refs. [14,15].

\begin{tabular}{lccc}
\hline \hline Decay mode & Predictions [keV] & {$[14][\mathrm{keV}]$} & {$[15][\mathrm{keV}]$} \\
\hline$\Sigma_{c}^{*++} \rightarrow \Sigma_{c}^{++} \gamma$ & $12 r_{1}^{2}$ & $\ldots$ & 11.6 \\
$\Sigma_{c}^{*+} \rightarrow \Sigma_{c}^{+} \gamma$ & $0.75 r_{1}^{2}$ & $\ldots$ & 0.85 \\
$\Sigma_{c}^{* 0} \rightarrow \Sigma_{c}^{0} \gamma$ & $3.1 r_{1}^{2}$ & $\ldots$ & 2.92 \\
$\Lambda_{c 1}^{*+} \rightarrow \Lambda_{c 1}^{+} \gamma$ & $0.13 r_{1}^{2}$ & $0.107 c_{R}^{2}$ & $\ldots$ \\
$\Sigma_{b}^{*+} \rightarrow \Sigma_{b}^{+} \gamma$ & $0.42 r_{1}^{2}$ & $\ldots$ & 0.60 \\
$\Sigma_{b}^{* 0} \rightarrow \Sigma_{b}^{0} \gamma$ & $0.024 r_{1}^{2}$ & $\ldots$ & 0.02 \\
$\Sigma_{b}^{*-} \rightarrow \Sigma_{b}^{-} \gamma$ & $0.089 r_{1}^{2}$ & $\ldots$ & 0.06 \\
$\Lambda_{b 1}^{* 0} \rightarrow \Lambda_{b 1}^{0} \gamma$ & $0.0013 r_{1}^{2}$ & $\ldots$ & $\ldots$ \\
\hline \hline
\end{tabular}

$$
\begin{gathered}
C_{\Sigma_{c}^{*+} \Sigma_{c}^{++} \gamma}=C_{\Sigma_{b}^{*+} \Sigma_{b}^{+} \gamma}=\frac{2}{3}, \\
C_{\Sigma_{c}^{*+} \Sigma_{c}^{+} \gamma}=C_{\Sigma_{b}^{* 0} \Sigma_{b}^{0} \gamma}=\frac{1}{6}, \\
C_{\Sigma_{c}^{* 0} \Sigma_{c}^{0} \gamma}=C_{\Sigma_{b}^{*-} \Sigma_{b}^{-} \gamma}=-\frac{1}{3}, \\
C_{\Lambda_{c 1}^{*+} \Lambda_{c 1}^{+} \gamma}=C_{\Lambda_{b}^{* 0} \Lambda_{b}^{0} \gamma}=-\frac{1}{6} .
\end{gathered}
$$

From this, one naively expects that ratios of radiative decay widths are determined from the squares of these constants as

$C_{\Sigma_{c}^{*++} \Sigma_{c}^{++} \gamma}^{2}: C_{\Sigma_{c}^{*+} \Sigma_{c}^{+} \gamma}^{2}: C_{\Sigma_{c}^{* 0} \Sigma_{c}^{0} \gamma}^{2}: C_{\Lambda_{c 1}^{*} \Lambda_{c 1} \gamma}^{2}=16: 1: 4: 1$

In Table X, we show our predictions on the decay widths of $\Lambda_{Q 1}^{*} \rightarrow \Lambda_{Q 1} \gamma$ and $\Sigma_{Q}^{*} \rightarrow \Sigma_{Q} \gamma$ comparing with the predictions in Refs. $[14,15]$. The predicted values for $\Sigma_{Q}^{*} \rightarrow \Sigma_{Q} \gamma$ decay widths are consistent with the ratio in Eq. (47), while the values for $\Lambda_{Q 1}^{*} \rightarrow \Lambda_{Q 1} \gamma$ decay widths are much smaller than the ratio in Eq. (47). This is because the mass

TABLE XI. Radiative decay widths of $\Lambda_{Q 1}^{(*)} \rightarrow \Sigma_{Q}^{(*)} \gamma$ in units of $\mathrm{keV}$. The values in the row indicated by "Predictions" are our predicted values, where $r_{2}$ is an undetermined parameter of $\mathcal{O}(1)$. For comparison, we list predictions in Ref. [14].

\begin{tabular}{lcc}
\hline \hline Decay mode & Predictions [keV] & {$[14][\mathrm{keV}]$} \\
\hline$\Lambda_{c 1}^{+} \rightarrow \Sigma_{c}^{+} \gamma$ & $250 r_{2}^{2}$ & $127 c_{R S}^{2}$ \\
$\Lambda_{c 1}^{+} \rightarrow \Sigma_{c}^{*+} \gamma$ & $21 r_{2}^{2}$ & $6 c_{R S}^{2}$ \\
$\Lambda_{c 1}^{*+} \rightarrow \Sigma_{c}^{+} \gamma$ & $120 r_{2}^{2}$ & $58 c_{R S}^{2}$ \\
$\Lambda_{c 1}^{*+} \rightarrow \Sigma_{c}^{*+} \gamma$ & $160 r_{2}^{2}$ & $54 c_{R S}^{2}$ \\
$\Lambda_{b 1}^{0} \rightarrow \Sigma_{b}^{0} \gamma$ & $98 r_{2}^{2}$ & $\ldots$ \\
$\Lambda_{b 1}^{0} \rightarrow \Sigma_{b}^{* 0} \gamma$ & $25 r_{2}^{2}$ & $\ldots$ \\
$\Lambda_{b 1}^{* 0} \rightarrow \Sigma_{b}^{0} \gamma$ & $31 r_{2}^{2}$ & $\ldots$ \\
$\Lambda_{b 1}^{* 0} \rightarrow \Sigma_{b}^{* 0} \gamma$ & $81 r_{2}^{2}$ & $\ldots$ \\
\hline \hline
\end{tabular}

TABLE XII. Radiative decay widths of $\Lambda_{Q 1}^{(*)} \rightarrow \Lambda_{Q} \gamma$ in units of $\mathrm{keV}$. The values in the row indicated by "Predictions" are our predicted values, where $r_{3}$ is an undetermined parameter of $\mathcal{O}(1)$. For comparison, we list predictions in Ref. [14].

\begin{tabular}{lcc}
\hline \hline Decay mode & Predictions [keV] & [14] [keV] \\
\hline$\Lambda_{c 1} \rightarrow \Lambda_{c} \gamma$ & $25 r_{3}^{2}$ & $191 c_{R T}^{2}$ \\
$\Lambda_{c 1}^{*} \rightarrow \Lambda_{c} \gamma$ & $35 r_{3}^{2}$ & $253 c_{R T}^{2}$ \\
$\Lambda_{b 1} \rightarrow \Lambda_{b} \gamma$ & $27 r_{3}^{2}$ & $\ldots$ \\
$\Lambda_{b 1}^{*} \rightarrow \Lambda_{b} \gamma$ & $29 r_{3}^{2}$ & $\ldots$ \\
\hline \hline
\end{tabular}

differences between $\Lambda_{Q 1}^{*}$ and $\Lambda_{Q 1}$ are quite small generating huge phase space suppression. We note that our predictions are consistent with the predictions in Refs. [14,15].

We next study the $\Lambda_{Q 1}^{(*)} \rightarrow \Sigma_{Q}^{(*)} \gamma$ decays which concern the $r_{2}$-term. The decay widths are expressed as

$$
\begin{aligned}
& \Gamma_{\Lambda_{Q 1} \rightarrow \Sigma_{Q \gamma}}=\frac{16 \alpha r_{2}^{2}}{9 F^{2}} \frac{m_{\Sigma_{Q}}}{m_{\Lambda_{Q 1}}} E_{\gamma}^{3}, \\
& \Gamma_{\Lambda_{Q 1} \rightarrow \Sigma_{Q}^{*} \gamma}=\frac{8 \alpha r_{2}^{2}}{9 F^{2}} \frac{m_{\Sigma_{Q}^{*}}}{m_{\Lambda_{Q 1}}} E_{\gamma}^{3}, \\
& \Gamma_{\Lambda_{Q 1}^{*} \rightarrow \Sigma_{Q \gamma}}=\frac{4 r_{2}^{2}}{9 F^{2}} \frac{m_{\Sigma_{Q}}}{m_{\Lambda_{Q 1}^{*}}} E_{\gamma}^{3}, \\
& \Gamma_{\Lambda_{Q 1}^{*} \rightarrow \Sigma_{Q}^{*} \gamma}=\frac{20 \alpha r_{2}^{2}}{9 F^{2}} \frac{m_{\Sigma_{Q}^{*}}}{m_{\Lambda_{Q 1}^{*}}} E_{\gamma}^{3} .
\end{aligned}
$$

In Table XI, we show our predictions comparing with those in Ref. [14].

The $r_{3}$-term generates the $\Lambda_{Q 1}^{(*)} \rightarrow \Lambda_{Q \gamma}$ decay, the width of which is expressed as

$$
\Gamma_{\Lambda_{Q 1}^{(*)} \rightarrow \Lambda_{Q \gamma} \gamma}=\frac{8 \alpha r_{3}^{2} f_{\pi}^{2}}{27 F^{4}} \frac{m_{\Lambda_{Q}}}{m_{\Lambda_{Q 1}^{(*)}}} E_{\gamma}^{3} .
$$

In Table XII, we show our predictions together with the ones in Ref. [14].

The width of $\Sigma_{Q}^{(*)} \rightarrow \Lambda_{Q} \gamma$ decay via the $r_{4}$-term is given by

TABLE XIII. Radiative decay widths of $\Sigma_{Q}^{(*)} \rightarrow \Lambda_{Q} \gamma$ in units of $\mathrm{keV}$. The values in the row indicated by "Predictions" are our predicted values, where $r_{4}$ is an undetermined parameter of $\mathcal{O}(1)$. For comparison, we list predictions in Ref. [15].

\begin{tabular}{lcc}
\hline \hline Decay mode & Predictions [keV] & {$[15][\mathrm{keV}]$} \\
\hline$\Sigma_{c}^{+} \rightarrow \Lambda_{c}^{+} \gamma$ & $43 r_{4}^{2}$ & 164 \\
$\Sigma_{c}^{*+} \rightarrow \Lambda_{c}^{+} \gamma$ & $110 r_{4}^{2}$ & 893 \\
$\Sigma_{b}^{0} \rightarrow \Lambda_{b}^{0} \gamma$ & $74 r_{4}^{2}$ & 288 \\
$\Sigma_{b}^{* 0} \rightarrow \Lambda_{b}^{0} \gamma$ & $99 r_{4}^{2}$ & 435 \\
\hline \hline
\end{tabular}




$$
\Gamma_{\Sigma_{Q}^{(*)} \rightarrow \Lambda_{Q} \gamma}=\frac{8 \alpha r_{4}^{2} f_{\pi}^{2}}{3 F^{4}} \frac{m_{\Lambda_{Q}}}{m_{\Sigma_{Q}^{(*)}}} E_{\gamma}^{3},
$$

and the predicted values are shown in Table XIII.

\section{A SUMMARY AND DISCUSSIONS}

We constructed an effective hadronic model regarding $\Lambda_{Q 1}=\left\{\Lambda_{c}\left(2595, J^{P}=1 / 2^{-}\right), \Lambda_{b}\left(5912,1 / 2^{-}\right)\right\}$and $\Lambda_{Q 1}^{*}=$ $\left\{\Lambda_{c}^{*}\left(2625,3 / 2^{-}\right), \Lambda_{b}^{*}\left(5920,3 / 2^{-}\right)\right\}$as chiral partners to $\Sigma_{Q}=\left\{\Sigma_{c}\left(2455,1 / 2^{+}\right), \Sigma_{b}\left(1 / 2^{+}\right)\right\}$and $\Sigma_{Q}^{*}=\left\{\Sigma_{c}^{*}(2520\right.$, $\left.\left.3 / 2^{+}\right), \Sigma_{b}^{*}\left(3 / 2^{+}\right)\right\}$, respectively, based on the chiral symmetry and heavy-quark spin-flavor symmetry. We determined the model parameters from the experimental data for relevant masses and decay widths of $\Sigma_{c}\left(2455,1 / 2^{+}\right)$, $\Sigma_{c}^{*}\left(2520,3 / 2^{+}\right)$, and $\Lambda_{c}\left(2595,1 / 2^{-}\right)$. Then, we studied the decay widths of $\Lambda_{c}^{*}(2625), \Lambda_{b}(5912)$, and $\Lambda_{b}(5920)$. We showed that the coupling constant for nonresonant contributions depicted in Figs. 1(c) and 1(e) is fixed from the $\Sigma_{c}^{(*)}$ decays reflecting the chiral partner structure. As a result, the decay of $\Lambda_{c}(2595)$ is dominated by the resonant contribution through $\Sigma_{c}(2455)$ depicted in Fig. 1(d), since the threshold of $\Lambda_{c}(2595) \rightarrow \Sigma_{c}^{+}(2455) \pi^{0}$ decay is open. We found nonresonant contributions depicted in Figs. 1(c) and 1(e) are comparable to resonant contributions for $\Lambda_{c}(2625), \Lambda_{b}(5912)$, and $\Lambda_{b}(5920)$, partly because the threshold for $\Sigma_{Q} \pi$ decays are not open. Our result indicates that studying nonresonant contributions will give a clue to understand the chiral partner structure for single heavy baryons.

We also studied the radiative decays of $\Sigma_{Q}^{(*)}$ and $\Lambda_{Q 1}^{(*)}$ using effective interaction Lagrangians in Eq. (44). We showed that there is a relation among $\Sigma_{Q}^{*} \rightarrow \Sigma_{Q} \gamma$ and $\Lambda_{Q 1}^{*} \rightarrow \Lambda_{Q 1} \gamma$ decays reflecting the chiral partner structure, which can be checked in future experiments.

In Table XIV, we summarize our predictions of the decay widths of single heavy baryons. We expect that comparison of these predictions with experimental data will give some clues to understand the chiral structure of single heavy baryons. We note that, since the hadronic decays of $\Lambda_{b 1}(5912)$ and $\Lambda_{b 1}^{*}(5920)$ are suppressed by the small phase space factors, radiative decays may be dominant modes.

Several comments are in order.

The present model does not include the decay $\Lambda_{Q 1} \rightarrow$ $\Lambda_{Q} \pi \pi$ via $\Sigma_{Q}^{*}$, which needs two pions in the $D$ wave in the heavy-quark limit. We expect that such decays are suppressed compared with the decays having two pions in the $S$ wave. Similarly, we expect that the decay $\Lambda_{Q 1}^{*} \rightarrow \Lambda_{Q} \pi \pi$ via $\Sigma_{Q}$ is also suppressed.

It is interesting to extend the present model including only two flavors to the one with the strange quark in addition
TABLE XIV. Predicted decay widths of SHBs. The row indicated by "Our Model" shows the predictions of the present analysis. The row indicated by "Expt." shows the experimental values for the full width of the relevant SHBs, in which "..." implies no experimental data.

\begin{tabular}{|c|c|c|c|c|}
\hline SHB & $J^{P} \mathrm{I}$ & Decay modes & s Our model $[\mathrm{MeV}]$ & Expt. $[\mathrm{MeV}]$ \\
\hline$\overline{\Sigma_{c}^{++}}$ & $1 / 2^{+}$ & $\Lambda_{c} \pi^{+}$ & $1.96_{-0.14}^{+0.07}$ & $1.89_{-0.18}^{+0.09}$ \\
\hline$\Sigma_{c}^{+}$ & $1 / 2^{+}$ & $\begin{array}{l}\Lambda_{c} \pi^{0} \\
\Lambda_{c} \gamma\end{array}$ & $\begin{array}{c}2.28_{-0.17}^{+0.09} \\
0.043 r_{4}^{2}\end{array}$ & $<4.6$ \\
\hline$\Sigma_{c}^{0}$ & $1 / 2^{+}$ & $\Lambda_{c} \pi^{-}$ & $1.94_{-0.14}^{+0.07}$ & $1.83_{-0.19}^{+0.11}$ \\
\hline$\Sigma_{c}^{*++}$ & $3 / 2^{+}$ & $\begin{array}{l}\Lambda_{c} \pi^{+} \\
\Sigma_{c}^{++} \gamma\end{array}$ & $\begin{array}{l}14.7_{-1.1}^{+0.6} \\
0.012 r_{1}^{2}\end{array}$ & $14.78_{-0.40}^{+0.30}$ \\
\hline$\Sigma_{c}^{*+}$ & $3 / 2^{+}$ & $\begin{array}{l}\Lambda_{c} \pi^{0} \\
\Sigma_{c}^{+} \gamma \\
\Lambda_{c} \gamma\end{array}$ & $\begin{array}{c}15.3_{-1.1}^{+0.6} \\
0.75 r_{1}^{2} \times 10^{-3} \\
0.11 r_{4}^{2}\end{array}$ & $<17$ \\
\hline$\Sigma_{c}^{* 0}$ & $3 / 2^{+}$ & $\begin{array}{c}\Lambda_{c} \pi^{-} \\
\Sigma_{c}^{0} \gamma\end{array}$ & $\begin{array}{c}14.7_{-1.1}^{+0.6} \\
3.1 r_{1}^{2} \times 10^{-3}\end{array}$ & $15.3_{-0.5}^{+0.4}$ \\
\hline$\Lambda_{c 1}$ & $1 / 2^{-}$ & $\begin{array}{c}\Lambda_{c} \pi^{+} \pi^{-} \\
\Lambda_{c} \pi^{0} \pi^{0} \\
\Sigma_{c}^{+} \gamma \\
\Sigma_{c}^{*+} \gamma \\
\Lambda_{c} \gamma\end{array}$ & $\begin{array}{c}0.562-1.09 \\
1.23-2.31 \\
0.25 r_{2}^{2} \\
0.021 r_{2}^{2} \\
0.025 r_{3}^{2}\end{array}$ & $2.59 \pm 0.30 \pm 0.47$ \\
\hline$\Lambda_{c 1}^{*}$ & $3 / 2^{-}$ & $\begin{array}{c}\Lambda_{c} \pi^{+} \pi^{-} \\
\Lambda_{c} \pi^{0} \pi^{0} \\
\Lambda_{c 1} \gamma \\
\Sigma_{c}^{+} \gamma \\
\Sigma_{c}^{*+} \gamma \\
\Lambda_{c} \gamma\end{array}$ & $\begin{array}{c}0.0618-0.507 \\
0.0431-0.226 \\
0.13 r_{1}^{2} \times 10^{-3} \\
0.12 r_{2}^{2} \\
0.16 r_{2}^{2} \\
0.035 r_{3}^{2}\end{array}$ & $<0.97$ \\
\hline$\Sigma_{b}^{+}$ & $1 / 2^{+}$ & $\Lambda_{b} \pi^{+}$ & $6.14_{-0.45}^{+0.23}$ & $9.7_{-2.8}^{+3.8+1.2}$ \\
\hline$\Sigma_{b}^{0}$ & $1 / 2^{+}$ & $\begin{array}{c}\Lambda_{b} \pi^{0} \\
\Lambda_{b}^{0} \gamma\end{array}$ & $\begin{array}{c}7.27_{-0.53}^{+0.27} \\
0.074 r_{4}^{2}\end{array}$ & 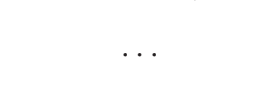 \\
\hline$\Sigma_{b}^{-}$ & $1 / 2^{+}$ & $\Lambda_{b} \pi^{-}$ & $7.02_{-0.51}^{+0.27}$ & $4.9_{-2.1}^{+3.1} \pm 1.1$ \\
\hline$\Sigma_{b}^{*+}$ & $3 / 2^{+}$ & $\begin{array}{c}\Lambda_{b}^{0} \pi^{+} \\
\Sigma_{b}^{+} \gamma\end{array}$ & $\begin{array}{c}11.0_{-0.8}^{+0.4} \\
0.42 r_{1}^{2} \times 10^{-3}\end{array}$ & $11.5_{-2.2}^{+2.7}+1.0$ \\
\hline$\Sigma_{b}^{* 0}$ & $3 / 2^{+}$ & $\begin{array}{l}\Lambda_{b} \pi^{0} \\
\Sigma_{b}^{+} \gamma \\
\Lambda_{b} \gamma\end{array}$ & $\begin{array}{c}12.3_{-0.9}^{+0.5} \\
0.024 r_{1}^{2} \times 10^{-3} \\
0.074 r_{4}^{2}\end{array}$ & $\ldots$ \\
\hline$\Sigma_{b}^{*-}$ & $3 / 2^{+}$ & $\begin{array}{c}\Lambda_{b} \pi^{-} \\
\Sigma_{b}^{-} \gamma \\
\Lambda_{b} \gamma\end{array}$ & $\begin{array}{c}11.9_{-0.9}^{+0.4} \\
0.089 r_{1}^{2} \times 10^{-3} \\
0.099 r_{4}^{2}\end{array}$ & $7.5_{-1.8}^{+2.2}+0.9$ \\
\hline$\Lambda_{b 1}$ & $1 / 2^{-}$ & $\begin{array}{c}\Lambda_{b} \pi^{+} \pi^{-} \\
\Lambda_{b} \pi^{0} \pi^{0} \\
\Sigma_{b}^{0} \gamma \\
\Sigma_{b}^{* 0} \gamma \\
\Lambda_{b} \gamma\end{array}$ & $\begin{array}{c}(0.67-4.4) \times 10^{-3} \\
(1.4-6.0) \times 10^{-3} \\
0.098 r_{2}^{2} \\
0.025 r_{2}^{2} \\
0.027 r_{3}^{2}\end{array}$ & $<0.66$ \\
\hline$\Lambda_{b 1}^{*}$ & $3 / 2^{-}$ & $\begin{array}{c}\Lambda_{b} \pi^{+} \pi^{-} \\
\Lambda_{b} \pi^{0} \pi^{0} \\
\Lambda_{b 1} \gamma \\
\Sigma_{b}^{0} \gamma \\
\Sigma_{b}^{* 0} \gamma \\
\Lambda_{b} \gamma\end{array}$ & $\begin{array}{c}(0.75-13) \times 10^{-3} \\
(2.2-12) \times 10^{-3} \\
0.0013 r_{1}^{2} \times 10^{-3} \\
0.031 r_{2}^{2} \\
0.081 r_{2}^{2} \\
0.029 r_{3}^{2}\end{array}$ & $<0.63$ \\
\hline
\end{tabular}


based on the chiral $\mathrm{SU}(3)_{L} \times \mathrm{SU}(3)_{R}$ symmetry. In this case, the flavor $\overline{3}$ representation including $\Lambda_{Q 1}$ becomes the chiral partner to the flavor 6 representation including $\Sigma_{Q}$. We leave the analysis for a future publication.

\section{ACKNOWLEDGMENTS}

This work was supported partly by JSPS KAKENHI Grant No. JP16K05345. We would like to thank Daiki Suenaga for useful discussions and comments.
[1] M. A. Nowak, M. Rho, and I. Zahed, Chiral effective action with heavy quark symmetry, Phys. Rev. D 48, 4370 (1993).

[2] M. A. Nowak and I. Zahed, Excited heavy mesons, Phys. Rev. D 48, 356 (1993).

[3] W. A. Bardeen and C. T. Hill, Chiral dynamics and heavy quark symmetry in a solvable toy field theoretic model, Phys. Rev. D 49, 409 (1994).

[4] W. A. Bardeen, E. J. Eichten, and C. T. Hill, Chiral multiplets of heavy-light mesons, Phys. Rev. D 68, 054024 (2003).

[5] M. A. Nowak, M. Rho, and I. Zahed, Chiral doubling of heavy light hadrons: BABAR 2317-MeV $/ c^{2}$ and CLEO 2463-MeV $/ c^{2}$ discoveries, Acta Phys. Polon. B 35, 2377 (2004).

[6] Y. L. Ma and M. Harada, Doubly heavy baryons with chiral partner structure, Phys. Lett. B 748, 463 (2015).

[7] Y. L. Ma and M. Harada, Degeneracy of doubly heavy baryons from heavy quark symmetry, Phys. Lett. B 754, 125 (2016).

[8] Y. L. Ma and M. Harada, Chiral partner structure of doubly heavy baryons with heavy quark spin-flavor symmetry, arXiv:1709.09746.

[9] M. A. Nowak, M. Praszalowicz, M. Sadzikowski, and J. Wasiluk, Chiral doublers of heavy light baryons, Phys. Rev. D 70, 031503 (2004).

[10] M. Harada and Y. L. Ma, Chiral partner structure of heavy baryons from the bound state approach with hidden local symmetry, Phys. Rev. D 87, 056007 (2013).

[11] Y. R. Liu and M. Oka, $\Lambda_{c} N$ bound states revisited, Phys. Rev. D 85, 014015 (2012).

[12] H. Nagahiro, S. Yasui, A. Hosaka, M. Oka, and H. Noumi, Structure of charmed baryons studied by pionic decays, Phys. Rev. D 95, 014023 (2017).

[13] A. J. Arifi, H. Nagahiro, and A. Hosaka, Three-body decay of $\Lambda_{c}^{*}(2595)$ and $\Lambda_{c}^{*}(2625)$ with consideration of $\Sigma_{c}(2455) \pi$ and $\Sigma_{c}^{*}(2520) \pi$ in intermediate states, Phys. Rev. D 95, 114018 (2017).

[14] P. L. Cho, Strong and electromagnetic decays of two new $\Lambda_{c}^{*}$ baryons, Phys. Rev. D 50, 3295 (1994).

[15] N. Jiang, X. L. Chen, and S. L. Zhu, Electromagnetic decays of the charmed and bottom baryons in chiral perturbation theory, Phys. Rev. D 92, 054017 (2015).
[16] C. Patrignani et al. (Particle Data Group), Review of particle physics, Chin. Phys. C 40, 100001 (2016).

[17] T. Aaltonen et al. (CDF Collaboration), Measurements of the properties of $\Lambda_{c}(2595), \Lambda_{c}(2625), \Sigma_{c}(2455)$, and $\Sigma_{c}(2520)$ baryons, Phys. Rev. D 84, 012003 (2011).

[18] R. Aaij et al. (LHCb Collaboration), Observation of Excited $\Lambda_{b}^{0}$ Baryons, Phys. Rev. Lett. 109, 172003 (2012).

[19] T. A. Aaltonen et al. (CDF Collaboration), Evidence for a bottom baryon resonance $\Lambda_{b}^{* 0}$ in CDF data, Phys. Rev. D 88, 071101 (2013).

[20] T. Yoshida, E. Hiyama, A. Hosaka, M. Oka, and K. Sadato, Spectrum of heavy baryons in the quark model, Phys. Rev. D 92, 114029 (2015).

[21] A. Hosaka, E. Hiyama, S. Kim, H.-C. Kim, H. Nagahiro, H. Noumi, M. Oka, K. Shirotori, T. Yoshida, and S. Yasui, Production and decay of charmed baryons, Nucl. Phys. A954, 341 (2016).

[22] H. Y. Cheng and C. W. Chiang, Quantum numbers of $\Omega_{c}$ states and other charmed baryons, Phys. Rev. D 95, 094018 (2017).

[23] K. L. Wang, Y. X. Yao, X. H. Zhong, and Q. Zhao, Strong and radiative decays of the low-lying $S$ - and $P$-wave singly heavy baryons, Phys. Rev. D 96, 116016 (2017).

[24] H. X. Chen, W. Chen, Q. Mao, A. Hosaka, X. Liu, and S. L. Zhu, $P$-wave charmed baryons from QCD sum rules, Phys. Rev. D 91, 054034 (2015).

[25] Q. Mao, H. X. Chen, W. Chen, A. Hosaka, X. Liu, and S. L. Zhu, QCD sum rule calculation for $P$-wave bottom baryons, Phys. Rev. D 92, 114007 (2015).

[26] H. X. Chen, Q. Mao, W. Chen, A. Hosaka, X. Liu, and S. L. Zhu, Decay properties of $P$-wave charmed baryons from light-cone QCD sum rules, Phys. Rev. D 95, 094008 (2017).

[27] H. Y. Cheng and C. K. Chua, Strong decays of charmed baryons in heavy hadron chiral perturbation theory: An update, Phys. Rev. D 92, 074014 (2015).

[28] H. X. Chen, W. Chen, X. Liu, Y. R. Liu, and S. L. Zhu, A review of the open charm and open bottom systems, Rep. Prog. Phys. 80, 076201 (2017).

[29] F. K. Guo, C. Hanhart, U. G. Meiner, Q. Wang, Q. Zhao, and B. S. Zou, Hadronic molecules, Rev. Mod. Phys. 90, 015004 (2018). 